\title{
Multiple unit roots in periodic autoregression
}

\author{
H. Peter Boswijk ${ }^{\mathrm{a}}$, Philip Hans Franses, ${ }^{*, b}$, Niels Haldrup ${ }^{\mathrm{c}}$ \\ 'Department of Economerrics, University of Amsterdam. Netherlands \\ 'Econametric Institute, Erasmus University. Rotterdam, P.O. Box 1738. \\ NL-3000 DR Rotrerdam, Netherlands \\ 'Depariment of Economics and Center for Nonlinear Modelling in Economics, Unizersity of Aarhus, \\ Denmark
}

Received December 1995; received in revised form July 1996

\begin{abstract}
In this paper we propose a model selection strategy for a univariate periodic autoregressive time series which involves tests for one or more unit roots and for parameter restrictions corresponding to seasonal unit roots and muttiple unit roots at the zero frequency. Examples of models that are considered are variants of the seasonal unit roots model and the periodic integration model. We show that the asymptotic distributions of various test statistics are the same as well-known distributions which are already tabulated. We apply our strategy to three empirical series to illustrate its ease of use. We find that evidence for seasonal unit roots based on nonperiodic models disappears when periodic representations are considered. (C) 1997 Elsevier Science S.A.
\end{abstract}

Key words: Periodic time series; Unit roots

JEL classification: C22

\section{Intruduction}

Periodic autoregressions (PAR) can yield useful descriptions of seasonally observed time series. Examples of their practical relevance for macroeconomic time series are given in Osborn (1988) and Franses and Paap (1994), among

\footnotetext{
- Corresponding author.
}

The first two authors thank the Royal Netherlands Academy of Arts and Sciences for its financial support. This paper was presented at the 7th World Meeting of the Econometric Society 1995 in Tokyo. Part of this paper was written while the third author visited the Department of Economics of the UC San Diego. The authors thank two anonymous referes for their helpful comments. 
many others. The key feature of PAR processes is that the AR parameters are allowed to vary with the season. This implies that the time series is a nonstationary time series since the autocovariance function varies with the season. This fact complicates the analysis of stochastic trends both at the zero frequency and at the so-called seasonal frequencies. To investigate unit root properties of PAR time series, it is therefore useful to write the PAR model in its multivariate form by stacking the seasonal time series into a vector of annual time series. In Franses (1994) it is proposed to apply the Johansen (1988) cointegration testing method to this vector process. This cointegration method can be used to test for nonseasonal and seasonal unit roots in a periodic time series, as well as to investigate the possibility of periodic integration, i.e. the usefulness of a differencing filter that varies with the season. The analysis of the multivariate representation of the PAR process does not impose all restrictions entailed by the univariate model, including seasonal homoskedasticity of the error process. Of course, such flexibility can lead to a reduction of empirical power in case the error process is not seasonally heteroskedastic, and hence may lead to the finding of too many unit roots, see, e.g. Franses and Romijn (1993). In the present paper, we propose a method to investigate multiple and seasonal unit roots imposing all the restrictions in the PAR process. Our approach extends the method in Boswijk and Franses (1996), where the presence of only a single unit root in a PAR process is studied.

We propose a model selection method for PAR processes which involves tests for unit roots at all frequencies of interest. The method can be easily applied in practice, since it only involves tests for the adequacy of certain nonlinear restrictions on the PAR parameters. An important advantage of our procedure is that no new tables with critical values have to be generated since the relevant asymptotic distributions are the same as those derived in Johansen $(1988,1991)$, for which several critical values are already tabulated in, e.g. Osterwald-Lenum (1992). Examples of models we consider are models where first and seasonal differences are assumed, the seasonal integration model and the periodic integration model. Hence, we also allow for processes where the differencing filter varies with the season. We extend the well-known HEGY procedure (Hylleberg et al., 1990), which concerns seasonal unit root processes in nonperiodic models. We also generalize the approach in Ghysels et al. (1996) by allowing for the possible presence of periodic differencing filters. An important feature of our method is that our generalization in fact amounts to simpler results in the sense that it does not require new asymptotic distributions.

The outline of our paper is as follows. In Section 2, we start off with a discussion of some preliminaries concerning notation and representation. In Section 3, we discuss the impact of multiple unit roots at the zero frequency. In Section 4, we propose our general-to-simple testing procedure for seasonal and nonseasonal unit roots. In Section 5, we evaluate our method through a Monte Carlo experiment. In Section 6, we apply our method to three illustrative 
quarterly time series. We show that the evidence for seasonal unit roots disappears when we allow for periodic variation in the AR parameters. Furthermore, we find that a periodic differencing filter is most useful to remove the stochastic trend from the data. In Section 7, we conclude this paper with some remarks.

\section{Some preliminaries}

In this paper we focus on a quarterly time series $y_{t}$ that can be described by a periodic autoregression of order $p(\operatorname{PAR}(p))$.

$$
y_{t}=\phi_{1 s} y_{t-1}+\cdots+\phi_{p s} y_{t-p}+\varepsilon_{t}, \quad t=1,2, \ldots, n, \quad s=1,2,3,4
$$

or

$$
\phi_{p, s}(B) y_{\mathrm{t}}=\varepsilon_{t},
$$

where $\phi_{i s}, i=1, \ldots, p$, are periodically varying parameters, $B$ is the backward shift operator, and where $\varepsilon_{1}$ is a standard white noise process. Although some of the $\phi_{p s}$ parameters can be equal to zero, and (1) allows for seasonally varying autoregressive lag lengths, we assume for the moment that $p$ is equal for all seasons. Furthermore, $\varepsilon_{t}$ is assumed to have nonseasonal variance. Note that, similar to the nonperiodic AR case, the value of $p$ restricts the number of unit roots in (1).

It can be argued that model (I) corresponds to a nonstationary process since the autocovariances of $y_{t}$ are not constant over time. In order to study unit root properties in $y_{r}$, it is therefore most convenient to rewrite (1) in vector notation. For example, for the PAR(2) process, one can write

$$
\begin{aligned}
{\left[\begin{array}{cccc}
1 & 0 & 0 & 0 \\
-\phi_{12} & 1 & 0 & 0 \\
-\phi_{23} & -\phi_{13} & 1 & 0 \\
0 & -\phi_{24} & -\phi_{14} & 1
\end{array}\right]\left[\begin{array}{l}
Y_{1 . T} \\
Y_{2 . T} \\
Y_{3 . T} \\
Y_{4 . T}
\end{array}\right]=} & {\left[\begin{array}{cccc}
0 & 0 & \phi_{21} & \phi_{11} \\
0 & 0 & 0 & \phi_{22} \\
0 & 0 & 0 & 0 \\
0 & 0 & 0 & 0
\end{array}\right]\left[\begin{array}{l}
Y_{1 . T-1} \\
Y_{2 . T-1} \\
Y_{3 . T-1} \\
Y_{4 . T-1}
\end{array}\right] } \\
& +\left[\begin{array}{l}
\varepsilon_{1 . r} \\
\varepsilon_{2, T} \\
\varepsilon_{3, T} \\
\varepsilon_{4, T}
\end{array}\right]
\end{aligned}
$$

where $Y_{s . T}$ and $\varepsilon_{s . T}$ are the observations on $y_{t}$ and $\varepsilon_{t}$ in season $s$ in year $T=1, \ldots, N=n / 4$, see Tiao and Grupe (1980) and Lütkepohl (199I), inter alia. This representation in (2) can be called a vector-of-quarters representation of order I (VQ(1)). Denoting $Y_{T}=\left(Y_{1, T}, \ldots, Y_{4, T}\right)^{\prime}$ and $\varepsilon_{T}=\left(\varepsilon_{1, T}, \ldots, \varepsilon_{4, T}\right)^{\prime},(2)$ can be summarized by

$$
\Phi_{0} Y_{T}=\Phi_{1} Y_{T-1}+\varepsilon_{T}
$$


Note that PAR processes with orders up to 4 can be written as VQ(1) processes. More generally, a $\operatorname{PAR}(p)$ process corresponds to a $\mathrm{VQ}(P)$ process with $P=[(p-1) / 4]+1$, where $[x]$ denotes integer part of $x$. Here we focus on (3) for notational convenience. To investigate the presence of unit roots in $y_{t}$ it is most convenient to check the solutions of the characteristic equation for (3), i.e.

$$
\left|\Phi_{0}-\Phi_{1} z\right|=0 .
$$

If one or more solutions to (4) correspond to $z=1$, then (4) can be expressed in error correction form, i.e.

$$
\Delta Y_{T}=\Pi Y_{T-1}+v_{T},
$$

where $v_{T}=\Phi_{0}^{-1} \varepsilon_{T}$, where

$$
\Pi=\Phi_{0}^{-1} \Phi_{1}-I,
$$

and where $\Delta=(1-B)$ denotes the first-order differencing filter so that $\Delta Y_{T}=Y_{T}-Y_{T-1}$.

Franses (1994) considers testing for (multiple) unit roots using the Johansen (1988) method applied to (5), without imposing the restrictions implied by the original model (1) on $\Pi$ and $\Omega$, the covariance matrix of $v_{\mathrm{t}}$. Suppose for example that $p=4$. In that case the original model has $16+1$ parameters $\left(\left\{\phi_{i s}\right\}, \sigma^{2}\right)$, whereas the unrestricted $V Q(1)$ model has $16+10$ unrestricted parameters in $(\Pi, \Omega)$. The nature of the 9 restrictions implied by the PAR(4) is most easily analyzed by transforming (5) in recursive form:

$$
A_{0} \Delta Y_{T}=A_{1} Y_{\mathrm{T}-1}+\eta_{T},
$$

where $A_{0}$ is a lower-triangular matrix with unit elements on the main diagonal, such that $A_{0} \Omega A_{0}^{\prime}=\operatorname{diag}\left(\sigma_{1}^{2}, \ldots, \sigma_{4}^{2}\right)$, and hence that $\eta_{T}=A_{0} v_{T}$ has a diagonal covariance matrix, and where $A_{1}=\dot{A}_{0} \Pi$. It is easily seen that the PAR(4) nociel implies $A_{0}=\Phi_{0}, A_{1}=\Phi_{1}-\Phi_{0}$ and $\sigma_{s}^{2}=\sigma^{2}$ for $s=1,2,3,4$. Thus, three of the nine restrictions correspond to periodic homoskedasticity, and the other 6 are restrictions across $A_{0}$ and $A_{1}$ (or, in the original form, across $\Pi$ and $\Omega$ ). Of course, when $p$ is smaller than 4 , there will he even more restrictions. The purpose of this paper is to obtain a possible power gain from imposing these restrictions, i.e. by testing for unit roots directly in (1) instead of in the unrestricted VQ model.

\section{Periodic autoregression integrated of order 2}

If there is one unity solution to (4), Boswijk and Franses (1996) show that any $\operatorname{PAR}(p)$ process can be written as

$$
\psi_{p-1, s}(B)\left(1-\alpha_{s} B\right) y_{t}=\varepsilon_{1},
$$


where $\psi_{p-1 . s}(B)$ is a periodic autoregressive polynomial of order $p-1$ and $\alpha_{1} \alpha_{2} \alpha_{3} \alpha_{4}=1$. In VQ notation, this becomes

$$
\begin{aligned}
& \Psi(B) Y_{T}^{*}=\varepsilon_{T}, \\
& Y_{T}^{*}=\left(\Xi_{0}-\Xi_{1} B\right) Y_{T},
\end{aligned}
$$

where

$$
\Xi_{0}=\left[\begin{array}{rrrr}
1 & 0 & 0 & 0 \\
-\alpha_{2} & 1 & 0 & 0 \\
0 & -\alpha_{3} & 1 & 0 \\
0 & 0 & -\alpha_{4} & 1
\end{array}\right] \text { and } \Xi_{1}=\left[\begin{array}{llll}
0 & 0 & 0 & \alpha_{1} \\
0 & 0 & 0 & 0 \\
0 & 0 & 0 & 0 \\
0 & 0 & 0 & 0
\end{array}\right] \text {, }
$$

and where $\Psi(B)=\Psi_{0}-\Psi_{1} B-\cdots-\Psi_{h} B^{h}$, with $h=[(p-2) / 4]+1$.

It will be useful to start with the case $p=2$, and then generalize the results to higher-order models. In that case, $(8)$ reduces to

$$
\left(1-\beta_{s} B\right)\left(1-\alpha_{s} B\right) y_{r}=\varepsilon_{s}, \quad s=1, \ldots, 4,
$$

or, since $B$ also operates on $\alpha_{s}$, as

$$
y_{t}=\alpha_{s} y_{t-1}+\beta_{s}\left(y_{t-1}-\alpha_{s-1} y_{t-2}\right)+\varepsilon_{r},
$$

where $\alpha_{0}=\alpha_{4}$, and the $\Psi(B)$ polynomial in (9) can then be written as

$$
\Psi_{0}-\Psi_{1} B=\left[\begin{array}{rrrc}
1 & 0 & 0 & -\beta_{1} B \\
-\beta_{2} & 1 & 0 & 0 \\
0 & -\beta_{3} & 1 & 0 \\
0 & 0 & -\beta_{4} & 1
\end{array}\right]
$$

Hence, in terms of the $\alpha_{s}$ and $\beta_{s}$ coefficients, the VQ process $Y_{\gamma}$ reads

$$
\left(\Psi_{0}-\Psi_{1} \dot{B}\right)\left(\Xi_{0}-\Xi_{1} B\right) Y_{T}=\left(\Gamma_{0}-\Gamma_{1} B\right) Y_{T}=\varepsilon_{T}
$$

with $\Gamma_{0}$ and $\Gamma_{1}$ defined as

$$
\begin{aligned}
\Gamma_{0} & =\left[\begin{array}{cccc}
1 & 0 & 0 & 0 \\
-\left(\alpha_{2}+\beta_{3}\right) & 1 & 0 & 0 \\
\alpha_{2} \beta_{3} & -\left(\alpha_{3}+\beta_{3}\right) & 1 & 0 \\
0 & \alpha_{3} \beta_{4} & -\left(\alpha_{4}+\beta_{4}\right) & 1
\end{array}\right] \text { and } \\
\Gamma_{1} & =\left[\begin{array}{llcc}
0 & 0 & -\alpha_{4} \beta_{1} & \alpha_{1}+\beta_{1} \\
0 & 0 & 0 & -\alpha_{1} \beta_{2} \\
0 & 0 & 0 & 0 \\
0 & 0 & 0 & 0
\end{array}\right] .
\end{aligned}
$$

The characteristic equation of the polynomial in (13) can now be written as

$$
\left|\Gamma_{0}-\Gamma_{1} z\right|=\left(1-\alpha_{1} \alpha_{2} \alpha_{3} \alpha_{4} z\right)\left(1-\beta_{1} \beta_{2} \beta_{3} \beta_{4} z\right)=0 \text {. }
$$


In Boswijk and Franses (1996) it is assumed that $\beta_{1} \beta_{2} \beta_{3} \beta_{4}<1$. In the present paper, we relax this assumption and consider the case where there can be more than a single unit root.

As in (5), the VQ process in (13) can be written in error correction form like

$$
\Delta Y_{T}=\left(\Gamma_{0}^{-1} \Gamma_{1}-I_{4}\right) Y_{T-1}+\Gamma_{0}^{-1} \varepsilon_{T}=\Pi Y_{T-1}+\Gamma_{0}^{-1} \varepsilon_{T}
$$

Under the assumption of two unity solutions to the characteristic equation (15), i.e. $\alpha_{1} \alpha_{2} \alpha_{3} \alpha_{4}=\beta_{1} \beta_{2} \beta_{3} \beta_{4}=1$, the $\Pi$ matrix in (16) reduces to

$$
\Pi=\left[\begin{array}{rrcc}
-1 & 0 & -\alpha_{4} \beta_{1} & \alpha_{1}+\beta_{1} \\
0 & -1 & -\alpha_{4} \beta_{1}\left(\alpha_{2}+\beta_{2}\right) & \alpha_{1} x_{2}+\alpha_{2} \beta_{1}+\beta_{1} \beta_{2} \\
0 & 0 & -\kappa & \kappa / \alpha_{4} \\
0 & 0 & -\alpha_{4} \kappa & \kappa
\end{array}\right],
$$

with

$$
\kappa=\left(\alpha_{4} / \beta_{4}\right)\left(1+\left(\alpha_{3} / \beta_{3}\right)\left(1+\left(\alpha_{2} / \beta_{2}\right)\left(1+\left(\alpha_{1} / \beta_{1}\right)\right)\right)\right) .
$$

It can be seen from (17) and (18) that the rank of $\Pi$ will usually equal 3 , and hence that $\Pi$ can be written as $\Pi=\gamma \lambda^{\prime}$, where $\gamma$ and $\lambda$ are both of dimension $(4 \times 3)$. Consider for instance the case where $\alpha_{s}=\beta_{s}=1$ for all $s=1,2,3,4$. In this case the four $Y_{\text {s.T }}$ series are $I(2)$, see e.g. Haldrup (1994a, b) and Johansen (1992a).

When the restriction $\alpha_{1} \alpha_{2} \alpha_{3} \alpha_{4}=\beta_{1} \beta_{2} \beta_{3} \beta_{4}=1$ holds, there is another possibility. When $\kappa=0$ the $I$ matrix is of rank 2 . This may occur for several possible parameter configurations, but in particular when $\alpha_{s}=-\beta_{s}=1$ for all $s$. In this case the PAR(2) process reduces to $\left(1-B^{2}\right) y_{t}=\varepsilon_{l}$, and this implies the presence of one nonseasonal unit root and one seasonal unit root at the bi-annual frequency, see, e.g. Hylleberg et al. (1990). We shall return to this situation in the next section. In this section we will elaborate on the $I(2)$ case.

One further insight of a periodically doubly integrated time series can be obtained from considering the vector moving average (VMA) representation. From (11), define the series $y_{\mathrm{t}}^{*}=\left(1-\alpha_{s} B\right) y_{t}$ such that

$$
\left(1-\beta_{s} B\right) y_{t}^{*}=\varepsilon_{t} \text {. }
$$

It follows from Boswijk and Franses (1996) that the VQ representation of $y_{t}^{*}$ can be described as

$$
\Delta Y_{T}^{*}=\left(\Theta_{0}^{*}+\Theta_{1}^{*} B\right) \varepsilon_{T},
$$

where

$$
\Theta_{0}^{*}=\left[\begin{array}{cccc}
1 & 0 & 0 & 0 \\
\beta_{2} & 1 & 0 & 0 \\
\beta_{2} \beta_{3} & \beta_{3} & 1 & 0 \\
\beta_{2} \beta_{3} \beta_{4} & \beta_{3} \beta_{4} & \beta_{4} & 1
\end{array}\right]
$$


and

$$
\Theta_{1}^{*}=\left[\begin{array}{cccc}
0 & \beta_{3} \beta_{4} \beta_{1} & \beta_{4} \beta_{1} & \beta_{1} \\
0 & 0 & \beta_{4} \beta_{1} \beta_{2} & \beta_{1} \beta_{2} \\
0 & 0 & 0 & \beta_{1} \beta_{2} \beta_{3} \\
0 & 0 & 0 & 0
\end{array}\right] .
$$

If we denote $\left(\Theta_{0}^{*}+\Theta_{1}^{*} B\right) \varepsilon_{T}=u_{T}$, it follows equivalently that

$$
A^{2} Y_{T}=\left(\Theta_{0}+\Theta_{1} B\right) u_{T}=\left(\Theta_{0}+\Theta_{1} B\right)\left(\Theta_{0}^{*}+\Theta_{1}^{*} B\right) \varepsilon_{T},
$$

with $\Theta_{0}$ and $\theta_{1}$ defined as

$$
\Theta_{0}=\left[\begin{array}{cccc}
1 & 0 & 0 & 0 \\
\alpha_{2} & 1 & 0 & 0 \\
\alpha_{2} \alpha_{3} & \alpha_{3} & 1 & 0 \\
\alpha_{2} \alpha_{3} \alpha_{4} & \alpha_{3} \alpha_{4} & \alpha_{4} & 1
\end{array}\right]
$$

and

$$
\Theta_{1}=\left[\begin{array}{cccc}
0 & \alpha_{3} \alpha_{4} \alpha_{1} & \alpha_{4} \alpha_{1} & \alpha_{1} \\
0 & 0 & \alpha_{4} \alpha_{1} \alpha_{2} & \alpha_{1} \alpha_{2} \\
0 & 0 & 0 & \alpha_{1} \alpha_{2} \alpha_{3} \\
0 & 0 & 0 & 0
\end{array}\right]
$$

and hence the characteristic equation of the VMA polynomial can be described as

$$
\begin{aligned}
|\theta(z)| & =\left|\Theta_{0}+\Theta_{1} z\right|\left|\Theta_{0}^{*}+\Theta_{1}^{*} z\right| \\
& =\left(1-\alpha_{1} \alpha_{2} \alpha_{3} \alpha_{4} z\right)^{3}\left(1-\beta_{1} \beta_{2} \beta_{3} \beta_{4} z\right)^{3}=(1-z)^{6}=0,
\end{aligned}
$$

when $\alpha_{1} \alpha_{2} \alpha_{3} \alpha_{4}=1$ and $\beta_{1} \beta_{2} \beta_{3} \beta_{4}=1$. Since the $\Delta^{2}$ filter applied to the VQ series $Y_{T}$ induces 8 unit roots, it can therefore be observed that only 2 of these will be in common, i.e. a single doubly integrated process driving the system.

All of the above representational results are easily generalized to the case where $p>2$. In that case, write the model as

$$
\lambda_{p-2, s}(B)\left(1-\beta_{s} B\right)\left(1-\alpha_{s} B\right) y_{1}=\varepsilon_{t},
$$

where $\lambda_{p-2, s}(B)$ is a periodic autoregressive polynomial of order $p-2$, In VQ notation this becomes

$$
A(B)\left[\Psi_{0}-\Psi_{1} B\right]\left[\Xi_{0}-\Xi_{1} B\right] Y_{T}=\varepsilon_{T},
$$

where $A(B)$ is a matrix lag polynomial of order $[(p+1) / 4]$. If the characteristic equation $\left|A(z)\left(\Psi_{0}-\Psi_{1} z\right)\left(\Xi_{0}-\Xi_{1} z\right)\right|=0$ has only two unit roots and all other roots outside the unit circle, then $A(B)$ is invertible, leading to

$$
\left[\Psi_{0}-\Psi_{1} B\right]\left[\Xi_{0}-\Xi_{1} B\right] Y_{T}=[A(B)]^{-1} \varepsilon_{\gamma} \text {. }
$$


Hence, the above results carry through after replacing $\varepsilon_{T}$ by $[A(B)]^{-1} \varepsilon_{T}$, a periodically stationary process. For further analysis we need to derive the limiting behavior of the $Y_{T}$ process.

Lemma 3.1. Let $Y_{T}$ be generated according to the PAR( $p$ ) process (25), or alternatively,

$$
\Delta^{2} Y_{T}=\left(\Theta_{0}+\Theta_{1} B\right)\left(\Theta_{0}^{*}+\Theta_{1}^{*} B\right) \Lambda(B)^{-1} \varepsilon_{T},
$$

where $A(B)$ is invertible, $\left\{\varepsilon_{\tau}\right\}$ i.i.d. $\mathrm{N}\left(0, \sigma^{2} I_{4}\right)$ and where $\Theta_{0}, \Theta_{1}, \Theta_{0}^{*}$ and $\Theta_{1}^{*}$ are defined in (21) and (23) with $\alpha_{1} \alpha_{2} \alpha_{3} \alpha_{4}=\beta_{1} \beta_{2} \beta_{3} \beta_{4}=1$, and $\kappa$ in (18) is not equal to 0 . Then we have as $N \rightarrow \infty$,

$$
\begin{aligned}
& N^{-3 / 2} Y_{[r N]} \stackrel{\mathrm{d}}{\rightarrow} \bar{B}(r)=w a \int_{0}^{r} W(u) \mathrm{d} u, \quad r, u \in[0,1] \\
& N^{-1 / 2} Y_{[r N]}^{*} \stackrel{\mathrm{d}}{\rightarrow} B^{*}(r)=\omega^{*} a^{*} W(r),
\end{aligned}
$$

where $[r N]$ denotes the integer part of $r N$, where $B(r)$ and $B^{*}(r)$ are $(4 \times 1)$ vector Brownian motion processes with covariance matrices $\omega^{2} a a^{\prime}$ and $\omega^{* 2} a^{*} a^{* \prime}, W(r)$ is a standard (scalar) Brownian motion process, and

$$
\begin{aligned}
\omega & =\sigma\left(b^{\prime} a^{*}\right)\left\{b^{* \prime} A(1)^{-1} A(1)^{-1} b^{*}\right\}^{1 / 2}, \\
\omega^{*} & =\sigma\left\{b^{* \prime} A(1)^{-1} A(1)^{\prime-1} b^{*}\right\}^{1 / 2},
\end{aligned}
$$

with

$$
a=\left[\begin{array}{c}
1 \\
\alpha_{2} \\
\alpha_{2} \alpha_{3} \\
\alpha_{2} \alpha_{3} \alpha_{4}
\end{array}\right], \quad b=\left[\begin{array}{c}
1 \\
\alpha_{1} \alpha_{3} \alpha_{4} \\
\alpha_{1} \alpha_{4} \\
\alpha_{1}
\end{array}\right], \quad a^{*}=\left[\begin{array}{c}
1 \\
\beta_{2} \\
\beta_{2} \beta_{3} \\
\beta_{2} \beta_{3} \beta_{4}
\end{array}\right] \text { and } b^{*}=\left[\begin{array}{c}
1 \\
\beta_{1} \beta_{3} \beta_{4} \\
\beta_{1} \beta_{4} \\
\beta_{1}
\end{array}\right] \text {. }
$$

Proof: see Appendix.

\subsection{Quasi-differencing an $1(2)$ process}

When testing for multiple unit roots in nonperiodic models Pantula (1989) suggests a sequential testing procedure. Assuming that at least a single unit root exists, the differenced time series is tested for the presence of an additional unit root. A similar strategy will be suggested here for periodic AR models. First, one should transform the $y_{t}$ series such that the resulting time series $y_{t}^{*}$ has $Y_{s, T}^{*}$ components that are at most $I(I)$. In the second step one can check whether $y_{i}^{*}$ has multiple unit roots, i.e. nonseasonal and/or seasonal unit roots. New 
problems arise for periodic models, however, since the way the time series should be transformed in the first step involves quasi-differencing by filters that are unknown and hence have to be estimated. The next theorem suggests how the quasi-difierencing filters across the seasons can be obtained.

Theorem 3.1. Let $y_{i}$ be a $\operatorname{PAR}(p)$ process satisfying the conditions stated in the Lemma 3.1, and consider NLS estimation of the PAR(2) model

$$
y_{t}=\sum_{s=1}^{4} \alpha_{s} D_{s, t} y_{t-1}+\sum_{s=1}^{4} \beta_{s} D_{s, t}\left(y_{t-1}-\alpha_{s-1} y_{t-2}\right)+\eta_{t},
$$

under the restriction $\alpha_{1} \alpha_{2} \alpha_{3} \alpha_{4}=1, t=1,2, \ldots, n$, where $D_{s, r}$ are the usual seasonal dummy variables and where $\alpha_{-k}=\alpha_{4-k}$, then it follows that

$$
\begin{aligned}
& N^{2}\left(\hat{\alpha}_{s}-\alpha_{s}\right)=O_{p}(1) \\
& N\left(\hat{\beta}_{s}-\beta_{s}\right)=O_{p}(1)
\end{aligned}
$$

for $s=1,2,3,4$.

Proof. See Appendix.

The significance of Theorem 3.1 is that in order to exclude the possibility of periodic models integrated of order 2 , initial estimates of the periodic coefficients in the periodic differencing filter $\left(1-\alpha_{s} B\right)$ can be obtained by a nonlinear regression. The auxiliary regression only needs to be of second order, regardless of the actual order of the PAR. Provided that the underlying time series is periodically doubly integrated a 'super-super' $\left(O_{p}\left(N^{2}\right)\right)$ consistent estimate of the periodic coefficients at the first order of integration can be calculated. These estimates form the basis for quasi-difierencing the time series. Next, the transformed series can be analyzed in accordance with the guidelines suggested below in Section 4.

\section{Model selection}

In this section we start with an analysis of $y_{t}$ time series with $Y_{s, T}$ series that are at most I(1). For such a series we propose test statistics for the number of unit roots in the $Y_{T}$ process, and tests for parameter restrictions that correspond with seasonal and nonseasonal unit roots. Furthermore, we discuss the impact of trends and constants on the asymptotic distributions of the test statistics and on the time series pattern under the various null hypotheses. Finally, we briefly discuss multiple trit roots in so-called subset PAR models, i.e. the AR order varies with the seasons. 
When the $Y_{s, T}$ processes are at most $I(1)$, there are five possible cases: the $(4 \times 1)$ vector process $Y_{T}$ contains $0,1,2,3$, or 4 unit roots, and hence $4,3,2,1$, and 0 cointegrating relationships. We consider each cast and derive the cointegrating vectors. These vectors imply nonlinear restrictions on the parameters in a $\operatorname{PAR}(p)$ process, which can easily be investigated using NLS techniques applied to the $\operatorname{PAR}(p)$ process

$$
\phi_{p, s}(B) y_{t}=\varepsilon_{\mathrm{r}} .
$$

\subsection{A single unit root}

The case of a single unit root is covered in Boswijk and Franses (1996). One unit root in $Y_{T}$ implies three cointegrating relationships, which can be expressed as

$$
\begin{aligned}
& Y_{4 . T}-\alpha_{4} Y_{3 . T} \\
& Y_{3 . T}-\alpha_{3} Y_{2 . T} \\
& Y_{2 . T}-\alpha_{2} Y_{1 . T} .
\end{aligned}
$$

These three relationships imply the fourth, i.e. $Y_{4 . T}-\alpha_{4} \alpha_{3} \alpha_{2} Y_{1 . T}$. Subtracting the stationary variable $\Delta Y_{4, \tau}$ and dividing by $-\alpha_{4} \alpha_{3} \alpha_{2}$ gives

$$
Y_{1, T}-\alpha_{1} Y_{4, T-1} \text { with } \alpha_{1} \alpha_{2} \alpha_{3} \alpha_{4}=1 \text {. }
$$

Given (32) and (33), the (periodically differenced) process $y_{t}-\alpha_{s} y_{t-1}$ under the restriction $x_{1} x_{2} \alpha_{3} x_{4}=1$ is a periodically stationary process. The PAR( $\left.p\right)$ process can then be written as

$$
\phi_{p . s}(B) y_{t}=\phi_{p-1 . s}(B)\left(1-\alpha_{s} B\right) y_{t}=\varepsilon_{\mathrm{r}} .
$$

Boswijk and Franses (1996) show that the likelihood ratio test

$$
\mathbf{L R}=n \log \left(\mathbf{R S S}_{\mathrm{r}} / \mathbf{R S S}_{\mathrm{u}}\right)
$$

for the hypothesis $\alpha_{1} \alpha_{2} \alpha_{3} \alpha_{4}=1$ in (34) follows the 'Johansen (1988) distribution' for rank 3 versus rank 4, where RSS, is the residual sum of squares [RSS] of (34) under the nonlinear restriction, and RSS $_{11}$ is the RSS of the unrestricted model. For further reference, we denote this test $L R_{1}$. In Boswijk and Franses (1996) it is also shown that, conditional on $\alpha_{1} \alpha_{2} \alpha_{3} \alpha_{4}=1$, the $F$-test for $\alpha_{s}=1, s=1,2,3$, follows a standard $F$-distribution. This seems confirmed for small samples by the simulation results in Franses and Paap (1994). Additionally, Boswijk and Franses (1996) show that a joint test for $\alpha_{s}=1$ in (34) follows a mixture of a Johansen-and an $F$-distribution. A drawback of the joint test is that when the null hypothesis $\alpha_{s}=1$ is rejected, the $y_{r}$ series may still have a stochastic trend in case of $\alpha_{1} \alpha_{2} \alpha_{3} \alpha_{4}=1$. Therefore, Buswijk and Franses (1996) advocate to use the 
two step approach. In the sequel of this section, we follow this strategy when investigating seasonal unit roots.

\subsection{Two unit roots}

In case of two unit roots in the $Y_{T}$ process, and still under the assumption that $Y_{T}$ is at most $I(1)$, there are two cointegrating relations between the $Y_{s, T}$ series, like, e.g.

$$
\begin{aligned}
& Y_{4 . T}-\beta_{14} Y_{3 . T}-\beta_{24} Y_{2, T} \\
& Y_{3 . T}-\beta_{13} Y_{2, T}-\beta_{23} Y_{1 . T} .
\end{aligned}
$$

These two relations imply two other cointegrating relationships

$$
\begin{aligned}
& Y_{2, T}-\beta_{12} Y_{1, T}-\beta_{22} Y_{4, T-1,} \\
& Y_{1, T}-\beta_{11} Y_{4, T-1}-\beta_{21} Y_{3, T-1}
\end{aligned}
$$

Since there are only two cointegrating relationships between the elements of $Y_{T}$, the relations in (38) and (39) should be linear combinations of those in (36) and (37). This implies the following four restrictions on the $\beta$ parameter values:

$$
\begin{aligned}
& \beta_{11}=-\beta_{13} / \beta_{23} \beta_{24}, \\
& \beta_{21}=\left(\left(1 / \beta_{23}\right)-\beta_{13} \beta_{14} /\left(\beta_{23} \beta_{24}\right)\right), \\
& \beta_{12}=-\beta_{14} \beta_{23} /\left(\beta_{13} \beta_{14}+\beta_{24}\right), \\
& \beta_{22}=1 /\left(\beta_{13} \beta_{14}+\beta_{24}\right) .
\end{aligned}
$$

These four parameter restrictions can be tested via imposing the corresponding nonlinear restrictions in the PAR $(p)$ model when it is rewritten as

$$
\phi_{p, s}(B) y_{t}=\phi_{p-2, s}(B)\left(1-\beta_{1 s} B-\beta_{2 s} B^{2}\right) y_{t}=\varepsilon_{t} .
$$

We denote the relevant likelihood ratio test statistic as $\mathbf{L R}_{2}$. We return with a discussion of its asymptotic distribution in Theorem 4.1 below.

In case the restrictions in (40) cannot be rejected, one may proceed with testing restrictions on the $\beta_{1 s}$ and $\beta_{2 s}$ values, which correspond to, e.g. certain seasonal and nonseasonal unit roots. Conditional on the restrictions in (40), one can construct likelihnod ratio statistics LR $_{2}^{*}$ for a particular hypothesis. Hence, $L_{2}^{*}$ for $(1-B)(1+B)$ in (41) implies that all $\beta_{1 s}=0$ and $\beta_{2 s}=1$. Imposing $\beta_{1 s}=0$ and $\beta_{2 s}=-1$ for all $s$ results in a $\left(1+B^{2}\right)$ filter, i.e. the seasonal unit roots $\pm i$. Note that when $\beta_{1 s}=2$ and $\beta_{2 s}=-1$, i.e. the case where the double filter $(1-B)^{2}$ is needed, and hence where $Y_{T}$ is at most $I(2)$, the restrictioss in (40) are violated. 


\subsection{Three unit roots}

In case there are three unit roots in the $Y_{T}$ process, there is only a single cointegrating relation between the $Y_{s, r}$ elements, which can be written as

$$
Y_{4, T}-\gamma_{14} Y_{3, T}-\gamma_{24} Y_{2, T}-\gamma_{34} Y_{1, T} \text {. }
$$

This relation implies three other cointegrating relationships, i.e.

$$
\begin{aligned}
& Y_{3 . T}-\gamma_{13} Y_{2 . T}-\gamma_{23} Y_{1, T}-\gamma_{33} Y_{4, T-1}, \\
& Y_{2 . T}-\gamma_{12} Y_{1 . T}-\gamma_{22} Y_{4 . T-1}-\gamma_{32} Y_{3 . T-1}, \\
& Y_{1 . T}-\gamma_{11} Y_{4 . T-1}-\gamma_{21} Y_{3 . T-1}-\gamma_{31} Y_{2, T-1} .
\end{aligned}
$$

Given (42), there are nine restrictions on the parameters in (43), i.e.

$$
\begin{array}{lll}
\gamma_{11} \gamma_{34}=1, & \gamma_{21} \gamma_{34}=-\gamma_{14}, & \gamma_{31} \gamma_{34}=-\gamma_{24}, \\
\gamma_{12} \gamma_{24}=-\gamma_{34}, & \gamma_{22} \gamma_{24}=1, & \gamma_{32} \gamma_{24}=-\gamma_{14}, \\
\gamma_{13} \gamma_{14}=-\gamma_{24}, & \gamma_{23} \gamma_{14}=-\gamma_{34}, & \gamma_{33} \gamma_{14}=1 .
\end{array}
$$

These restrictions can be tested in a rewritten version of (31), i.e.

$$
\phi_{p, s}(B) y_{t}=\phi_{p-3 . s}(B)\left(1-\gamma_{1 s} B-\gamma_{2 s} B^{2}-\gamma_{3 s} B^{3}\right) y_{t}=\varepsilon_{1} \text {. }
$$

We denote the likelihood ratio tes! statistic for the restrictions in (44) in (45) as LR. Similar to the case of two unit roots, and conditional on the restrictions (44), one may test for parameter restrictions as $(1-B)\left(1+B^{2}\right)$ in (45) using likelihood ratio test statistics $\mathbf{L R}_{3}^{*}$.

\subsection{Four unit roots}

In case of four unit roots, the general $\operatorname{PAR}(p)$ model can be written as

$$
\phi_{p, s}(B) y_{1}=\phi_{p-4, s}(B)\left(1-B^{4}\right) y_{t}=\varepsilon_{t} \text {. }
$$

The test for the hypothesis of four unit roots, which amounts to a linear restriction, will be denoted as $\mathbf{L R}_{\mathbf{4}}$.

Theorem 4.I. Under the hypothesis of $q$ unit roots, we have as $n \rightarrow \infty$,

$$
\operatorname{LR}_{q} \stackrel{\mathrm{d}}{\rightarrow} \operatorname{trace}\left\{\int_{0}^{1}\left(\mathrm{~d} W(s) W(s)^{\prime}\left(\int_{0}^{1} W(s) W(s)^{\prime} \mathrm{d} s\right)^{-1} \int_{0}^{1} W(s) \mathrm{d} W(s)^{r}\right\},\right.
$$

where $W(s)$ is a standard q-vector Brownian motion process.

Under the additional hypothesis of a nonperiodic (seasonal) unit root,

$$
\mathbf{L R}_{\boldsymbol{q}}^{*} \stackrel{d}{\rightarrow} \chi^{2}(k),
$$

where $k$ is the number of additional restrictions tested. 
Proof. See Appendix.

Notice that the limiting distribution of $\mathbf{L R}_{q}$ is the same as the one obtained and tabulated in Johansen (1988) for the likelihood ratio tests for $p-q$ cointegrating vectors (and hence $q$ unit roots) in a $p$-dimensional vector autoregressive process. Thus, the tests proposed here do not require new tables to be generated. This is in contrast to the approach followed in Ghysels et al. (1996), where it is proposed to test for the adequacy of, e.g. the $\left(1+B^{2}\right)$ filter within the general PAR( $p)$ model. The above theorem indicates that test statistics for such joint hypotheses asymptotically follow distributions that are complicated functions of Johansen- and $\chi^{2}$-distributions and, hence, that new critical values for those tesis have to be generated. An additional disadvantage is that rejection of the null hypothesis leaves open the question how many stochastic trends are driving the time series.

\subsection{Summary of our empirical procedure}

In practical occasions, the model selectior. strategy proceeds as follows. The first step is to estimate the order $p$ of the PAR process using some LR based test or one of the familiar information criteria. The simulation results in Franses and Paap (1994) indicate that the number of unit roots in the PAR process does not affect this order selection. In case one suspects $I(2)$ type patterns, one should estimate the $\alpha_{3}$ in a PAR(2) model, as suggested in Theorem 3.1. In a next step one can analyze the $y_{t}^{*}=\left(1-\hat{\alpha}_{s} B\right) y_{s}$ series for nonseasonal and seasonal unit roots via imposing nonlinear restrictions in decreasing sequence of unit roots and testing for the number of unit roots using our LR test statistics. Hence, the sequence is first to consider the LR $_{4}$ test. Finally, if the number of unit roots is determined, one may check for restrictions like $(1-B)$ or $(1+B)$ to investigate specific seasonal and nonseasonal unit roots.

\subsection{Constants and trends}

In many practical occasions, one may want to enlarge the model in (1) like

$$
y_{t}=\mu_{s}+\tau_{s} t+\phi_{1 s} y_{t-1}+\cdots+\phi_{p s} y_{t-p}+\varepsilon_{t}, \quad t=1,2, \ldots, n_{n}
$$

where $\mu_{s}$ are seesonally varying intercept terms and $\tau_{s}$ are seasonally varying parameters that correspond to the deterministic trcni. The inclusion of constants and trends in the regression model has an effect on the asymptotic distribution of the $L_{R}$ test statistics, $i=1,2,3,4$, see also the Appendix. The critical values of the relevant distributions are tabulated in Osterwald-Lenum (1992). 


\subsection{Subset PAR processes}

Until now we have assumed that the PAR process is of order $p$ sucil that the AR order is equal to $p$ for all seasons $s$. It may however occur in practice that the AR order in some season $s$, say $p_{s}$, is smaller than $p$. Furthermore, it may occur that $\phi_{j, s}$ parameters can be set equal to zero for some $j$ or $s$. In both cases, these models can be called subset PAR processes. Given the expressions for the characteristic equations for the $Y_{T}$ processes in Sectior: 2 , it is clear that the number of possible unit roots in a PAR process is determined by the minimum value of $p_{s}$. For example, for a $\operatorname{PAR}(2)$ as (11), the characteristic equation becomes $1-\alpha_{1} \alpha_{2} \alpha_{3} \alpha_{4} z=0$ when only a single $\beta_{s}$ value equals zero.

For practical purposes, we recommend that one first tests for unit roots before one checks whether the PAR model is a subset PAR model. This is because the distribution of $t$-test statistics for the significance of, for example, lagged $\left(1-\hat{\alpha}_{s} B\right) y_{t}$ variables depends on the number of (any remaining; stochastic trends.

\section{Monte Carlo simulations}

We now turn to a small-scale Monte Carlo experiment to assess the finite sample size and power properties of the tests proposed in the previous section, with a specific focus on the validity of the asymptotic results in Theorem 4.1. We consider 7 data generating processes [DGPs], all of which are special cases of the fourth-order periodic autoregression

$$
\phi_{4, s}(B) y_{t}=\mu_{s}+\varepsilon_{t},
$$

where in the DGPs we set $\mu_{s}$ at 0 for all $s$. The DGPs are

(DGP1)

$\phi_{4, s}(B)=\left(1+0.8 B+0.6 B^{2}+0.4 B^{3}\right)\left(1-\alpha_{s} B\right)$

with DGP1nopar: $\quad \alpha_{s}=1$ for all $s$

with DGP1par: $\quad \alpha_{s}=\{0.8,1,1.25,1\}$

(DGP2) $\quad \phi_{4, s}(B)=\left(1+0.6 B^{2}\right)\left(1-\alpha_{s} B^{2}\right)$

with DGP2nopar: $\quad \alpha_{s}=1$ for all $s$

with DGP2par: $\quad \alpha_{s}=\{0.8,1,1.25,1\}$

(DGP3) $\quad \phi_{4, s}(B)=(1+0.8 B)\left(1-\alpha_{s} B+B^{2}-\alpha_{s} B^{3}\right)$

with DGP3nopar: $\quad \alpha_{s}=1$ for all $s$

with DGP3par: $\quad \alpha_{s}=\{0.8,1.25,0.8,1.25\}$

(DGP4) $\quad \phi_{4, s}(B)=\left(1-B^{4}\right)$. 
Table I

Rejection frequencies of periodic unit root tests at a $\$ \%$ nominal level number of replications is 2000

\begin{tabular}{|c|c|c|c|c|c|}
\hline \multirow[b]{2}{*}{ DGP } & \multirow[b]{2}{*}{$n$} & \multicolumn{4}{|l|}{ Tests } \\
\hline & & $\mathbf{L R}_{\mathbf{t}}$ & $\mathbf{L R}_{2}$ & $\mathrm{LR}_{3}$ & $\mathbf{L R}_{4}$ \\
\hline \multirow[t]{2}{*}{ Inopar } & 100 & 0.037 & 0.274 & 0.577 & 0.851 \\
\hline & 200 & 0.049 & 0.948 & 1.000 & 1.000 \\
\hline \multirow[t]{2}{*}{$1 \mathrm{par}$} & 100 & 0.035 & 0.332 & 0.704 & 0.944 \\
\hline & 200 & 0.043 & 0.977 & 1.000 & 1.000 \\
\hline \multirow[t]{2}{*}{ 2nopar } & 100 & & 0.028 & 0.137 & 0.476 \\
\hline & 200 & & 0.038 & 0.699 & 0.993 \\
\hline \multirow[t]{2}{*}{ 2рат } & 100 & & 0.053 & 0.189 & 0.657 \\
\hline & 200 & & 0.044 & 0.791 & 1.000 \\
\hline \multirow[t]{2}{*}{ 3nopar } & 100 & & & 0.032 & 0.193 \\
\hline & 200 & & & 0.048 & 0.594 \\
\hline \multirow[t]{2}{*}{ 3раг } & 100 & & & 0.079 & 0.702 \\
\hline & 200 & & & 0.056 & 0.998 \\
\hline \multirow[t]{2}{*}{4} & 100 & & & & 0.073 \\
\hline & 200 & & & & 0.063 \\
\hline
\end{tabular}

Note that DGP $i$ involves $i$ unit roots, and that for $i=1,2,3$, DGPinopar is the nonperiodic model, and DGPipar is the periodic model. All periodicity in the DGPs is contained in the cointegrating linear combinations, and all short-run dynamics are nonperiodic. The construction of the tests, however, does not involve corresponding parameter restrictions. Furthermore, notice that the DGPs are chosen such that the characteristic roots of the VQ representation are the same for the periodic and nonperiodic DGPs.

Table 1 contains the rejection frequencies of the $L_{1}$ to $L_{2}$ statistics, for a sample size of 100 and 200 observations. All tests are based on the correct order of the PAR(4) model since the simulation results in Franses and Paap (1994) indicate that this order will usually be detected. The entries on the main diagonal of Table 1 represent the empirical size of the test, whereas the offdiagonal cells give the empirical power.

We do not compute rejection frequencies of the $\mathbf{Z} \mathbf{R}_{i}$ tests for $\mathrm{DGPj}$ with $j>i$ for two reasons. First, it is well known that if the DGP contains more unit roots than are tested, then the test will have a higher (asymptotic) rejection frequency than the nominal size. Thus, we should expect values exceeding 0.05 below the diagonal of Table 1, even asymptotically. Secondly, some of the parameters of the model under $i$ unit roots will not be identified when the DGP actually has more than $i$ unit roots. Therefore, convergence of NLS optimization methods will be problematic. The first problem can be solved by employing Johansen's (1992b) sequential testing procedure, based on the work by Pantula (1989). In this procedure, one starts with testing the maximum number of unit roots (in 
Table 2

Rejection frequencies of periodicity tests at a $5 \%$ nominal level, number of replications is 2000

\begin{tabular}{lllll}
\hline DGP & Test & $n$ & $\begin{array}{l}\text { Nonperiodic DGP } \\
\text { (size) }\end{array}$ & $\begin{array}{l}\text { Periodic DGP } \\
\text { (power) }\end{array}$ \\
\hline 1 & LR $_{1}^{*}$ & 100 & 0.092 & 0.219 \\
2 & & 200 & 0.071 & 0.556 \\
3 & RR $_{2}^{*}$ & 100 & 0.164 & 0.288 \\
& & 200 & 0.095 & 0.433 \\
& LR $_{3}^{*}$ & 100 & 0.253 & 0.364 \\
\hline
\end{tabular}

this case 4), and only proceeds to testing $i$ unit roots when the hypothesis of $i+1$ unit roots is rejected. This procedure will have an symptotically controllable size, see Johansen (1992b).

From Table 1, we observe that the empirical sizes are reasona! sly close to the nominal size of $5 \%$, and that the power of the tests seems to be higher for the periodic DGPs than for their nonperiodic counterparts.

Finally, in Table 2 we report the rejection frequencies of the $\mathbf{L R}^{*}$ tests for particular nonperiodic differencing filters, i.e. $(1-B)$ in DGP1, $\left(1-B^{2}\right)$ in DGP2 and $\left(1-B+B^{2}-B^{3}\right)=(1-B)\left(1+B^{2}\right)$ for DGP3. We observe from Table 2 that the finite sample size of the tests (i.e. the rejection frequencies for DGPinopar) can be quite far from $5 \%$, and seem to converge to the nominal size only slowly. Therefore, it may be worthwhile to investigate the effectiveness of, e.g. bootstrap methods or other small-sumple corrections for this testing problem. The power of the tests appear to increase with the sample size, as expected.

\section{Applications}

In this section we illustrate the empirical usefulness of our method to test for nonseasonal and seasonal unit roots in periodic autoregressions for three quarterly macroeconomic time series, which are selected for no particular reason other than illustrative purposes. These series are Unemployment Rate in Norway for 1966.1-1992.4 (not in logs), (Real) Consumption of Nondurables in the USA for 1947.1-1991.4 (in logs) and Unemployment in Canada for 1960.1-1987.4 (not in logs). We start our empirical analysis with an application of the HEGY test method for nonseasonal and seasonal unit roots in a nonperiodic AR model. Thie results are summarized in Table 3.

These results indicate that the nonseasonal unit root 1 is present for all three seriec. Unreported HEGY test results for the first-ori or dif $;$ is enced time series reveal that these series are at most $I(1)$ at the zero Irejuency. The scasonal unit 
Table 3

Testing for seasonal and nonseasonal unit roots in nonperiodic AR models using the HEGY method, where the auxiliary regression includes four seasonal dummies and a linear trend

\begin{tabular}{lccc}
\hline Tests $^{2}$ & $\begin{array}{l}\text { Unemployment } \\
\text { Norway }\end{array}$ & $\begin{array}{l}\text { Nondurables } \\
\text { Consumption ISA }\end{array}$ & $\begin{array}{l}\text { Unemployment } \\
\text { Canada }\end{array}$ \\
\hline$t\left(\pi_{1}\right)$ & -1.630 & -0.433 & -1.309 \\
$t\left(\pi_{2}\right)$ & $-2.626^{*}$ & -2.235 & -1.680 \\
$F\left(\pi_{3}, \pi_{4}\right)$ & $5.925^{*}$ & 5.061 & $7.155^{* *}$ \\
Lags & 2 & 6 & 5 \\
$n^{*}$ & 102 & 170 & 103 \\
\hline
\end{tabular}

"The test statistics, the relevant auxiliary regression and the appropriate critical values are given in Hylleberg et al. (1990). Lags denotes the number of lagged $y_{t}-y_{t-4}$ variables included in the auxiliary regression, and $n^{*}$ is the number of effective observations. The $t\left(\pi_{1}\right)$-test concerns the nonseasonal unit root 1 , the $t\left(\pi_{2}\right)$-test csncerns the seasonal unit root -1 , and the joint $F\left(\pi_{3}, \pi_{4}\right)$-test concerns the seasonal unit roots $\pm i$. The unit roots correspond with the $(1-B),(1+B)$ and $\left(1+B^{2}\right)$ differencing filters, respectively.

** Significant at the $1 \%$ level.

**Significant at the $5 \%$ level.

*Significant at the $10 \%$ level.

root -1 is present for all three series when we consider the $5 \%$ significance level. When we allow for the $10 \%$ significance level, we do not find this seasonal unit root to be present in unemployment in Norway. At the 5\% significance level, we obtain evidence for the seasonal unit roots $\pm i$ for $t w o$ of the three series. In sum, our three example series all have one or more seasonal unit roots in case we consider a nonperiodic model.

To investigate the robustness of the findings in Table 3 to periodicity in the AR parameters, we use the method proposed in Franses (1994). Within this method, the PAR model is allowed to have seasonally heteroskedastic error terms. The main results are presented in Table 4.

The Johansen type test statistics for the rank $r$ of the matrix $\Pi=\gamma \alpha^{\prime}$ in the VQ(1) model as in (5) indicate that this rank is equal to 1 for the two unemployment series and is equal to 2 or 3 for the consumption variable. These results clearly indicate that the rank is not equal to 0 , which corresponds to the adequacy of the $\left(1-B^{4}\right)$ transformation for $y_{t}$ (or the $\Delta$ transformation for $Y_{T}$ ). The value of 3 of the rank of $\Pi$ for the consumption series is clearly in contrast to the results in Table 3, where evidence for all four unit roots is reported. Since this cointegration-based method does not impose all restrictions implied by the PAR model, it will be less powerful than our new methods which do impose these restrictions, provided of course that these restrictions, such as seasonal homoskedasticity, are satisfied. 
Table 4

Testing for the cointegration rank $r$ of $\Pi=\gamma \alpha \alpha^{\prime}$ in the VQ(1) model (5) using the Johansen method

\begin{tabular}{lccc}
\hline Tests & $\begin{array}{l}\text { Unemployment } \\
\text { Norway }\end{array}$ & $\begin{array}{l}\text { Nondurables } \\
\text { Consumption USA }\end{array}$ & $\begin{array}{l}\text { Unemployment } \\
\text { Canada }\end{array}$ \\
\hline$\lambda_{1}$ & $0.973^{* * *}$ & $0.808^{* * * *}$ & $0.917^{* * * *}$ \\
$\lambda_{2}$ & 0.411 & $0.553^{* * *}$ & 0.415 \\
$\lambda_{3}$ & 0.248 & $0.263^{* *}$ & 0.260 \\
$\lambda_{4}$ & 0.088 & 0.037 & 0.044 \\
Trace $(r \leqslant 0)$ & $117.688^{* * *}$ & $123.023^{* * *}$ & $91.046^{* * *}$ \\
Trace $(r \leqslant 1)$ & 23.584 & $50.457^{* * *}$ & 23.813 \\
Trace $(r \leqslant 2)$ & 9.813 & 15.068 & 9.346 \\
Trace $(r \leqslant 3)$ & 2.392 & 1.641 & 1.223 \\
$N^{*}$ & 26 & 44 & 27 \\
Decision & $r=1$ & $r=3$ & $r=1$ \\
\hline
\end{tabular}

- The tests are the familiar $\lambda_{\max }$ and Trace test statistics, proposed in Johansen $(1988,1991)$, where $\lambda_{i}$ in the table refers to the relevant $\lambda_{\max }$ test. The asymptotic distribution of these tests is given in Osterwald-Lenum (1592). $N^{*}$ is the fffective sample size. Because of the small sample size, we use the critical values displayed in Franses (1994), for $N^{*}$ is 25 and 50 . Several model selection and diagnostic criteria indicate that the $\mathrm{YQ}(1)$ model sufficiently describes the annually observed vector time series $Y_{T}$.

*** Significant at the $1 \%$ level.

** Significant at the $5 \%$ level.

* Significant at the $10 \%$ level.

Our next step is to apply the LR tests which are proposed in Section 4 . The (unreported) HEGY outcomes for the $(1-B) y_{r}$ series suggest that $I(2)$ type behavior is not present in our three example series. Before we can apply our tests, we need to decide on the order of the various PAR models. Using diagnostic tests for residual autocorrelation at lags 1 and 1-4 and for periodic patterns in the residual autocorrelation function, we find that the order $p$ can be set at 4 for each time series. The residuals of the estimated PAR(4) model are also checked for the absence of seasonal heteroskedasticity. The $F$-test values of the auxiliary regression of $\hat{\varepsilon}_{t}^{2}$ on a constant and three seasonal dummies obtain the (5\%) insignificant values of $1.982,1.796$ and 2.407 . We also test whether the $A R$ parameters are indeed periodically varying. In the PAR(4) model, an $F$-test for the hypothesis of no periodicity follows a standard $\boldsymbol{F}$-distribution with 12 and $n-20$ degrees of freedom. See Boswijk and Franses (1996) for the derivation of this distribution. The test results for periodicity are $9.762,9.797$ and 3.102 , which indicate a convincing rejection of the null hypothesis of no periodic parameter variation for our three example series. 
Table 5

Testing for multiple unit roots in PAR(4) models using LR tests

\begin{tabular}{lccc}
\hline Tests & $\begin{array}{l}\text { Unemployment } \\
\text { Norway }\end{array}$ & $\begin{array}{l}\text { Nondurables } \\
\text { Consumption USA }\end{array}$ & $\begin{array}{l}\text { Unemployment } \\
\text { Canada }\end{array}$ \\
\hline LR $_{4}$ & $328.128^{* * *}$ & $196.432^{* * *}$ & $232.741^{* * *}$ \\
LR $_{3}$ & $123.700^{* * *}$ & $133.950^{* * *}$ & $164.240^{* * *}$ \\
LR $_{2}$ & $70.459^{* * *}$ & $64.315^{* * *}$ & $32.004^{* * *}$ \\
LR $_{1}$ & 0.469 & 2.056 & 1.040 \\
$\alpha_{1}$ & 1.014 & 1.053 & 1.077 \\
$\alpha_{2}$ & 1.057 & 1.018 & 0.982 \\
$\alpha_{3}$ & 1.012 & 1.022 & 0.971 \\
$\alpha_{4}$ & 0.922 & 0.913 & 0.974 \\
$F_{11-* 1}$ & $7.457^{* * *}$ & & $3.895^{* * *}$ \\
\hline
\end{tabular}

a The $\operatorname{LR}_{i}(i=1,2,3,4)$ test statistics are discussed in Section 4 . The $\alpha_{s}(s=1,2,3,4)$ estimates concern the parameters in the periodic differencing filter $\left(1-\alpha_{5} B\right)$ under the restriction $\alpha_{1} \alpha_{2} \alpha_{3} \alpha_{4}=1$, which transforms the $y_{t}$ series to periodic stationarity. The $F_{\left(t-n_{1}\right.}$ test concerns an $F$-test for the restriction $\alpha_{3}=1$, conditional on $\alpha_{1} \alpha_{2} \alpha_{3} \alpha_{4}=1$. This $F$-test has a standard $F$-distribution with 3 and $m$ degrees of freedom, with $m$ equal to $n-4-4 p+1$. The PAR(4) models contain 4 seasonal intercept terms, but no seasonal trends. The rosults do not change very much when these trends are included.

*** Significant at the $1 \%$ level.

** Signiticant at the $5 \%$ level.

* Significant at the $10 \%$ level.

The results for the LR test statistics for 1-4 unit roots are given in Table 5 . Compareo with Table 4, we may expect different findings given that our new method imposes all restrictions implied by the PAP(4) model (including seasonal homoskedasticity) and the diagnostic mentioned above suggest that these restrictions are valid.

The results for the $\operatorname{LR}_{i}(i=1,2,3,4)$ statistics in Table 5 can easily be summarized. Only the $\mathbf{L R}_{1}$ test value is insignificant, while the null hypotheses corresponding to the $L_{2}$ to $L_{R_{4}}$ tests are rejected at the $1 \%$ level (or even at the $0.1 \%$ level). Hence, there appears to be only a single unit root in each of the three time series considered. Finally, we investigate if this single stochastic trend can be removed using the $(1-B)$ filter. The values of the $F_{(1-B)}$ test for this hypothesis in the last row of Table 5 indicate a firm rejection. In other words, our three example series all seem periodically integrated of order 1 . This means that the appropriate differencing filter for these series is $\left(1-\alpha_{5} B\right)$ with $\alpha_{1} \alpha_{2} \alpha_{3} \alpha_{4}=1$, where the estimates of $\alpha_{3}$ are also given in Table 5 . 


\section{Concluding remarks}

In this paper we propose and apply a simple testing strategy for periodic autoregressions, which involves an investigation of seasonal unit roots and one or two nonseasonal unit roots. Our method also allows for periodic integration of order 1 and 2. The latter class of methods may yield useful descriptions of seasonal time series since it allows the seasonal fluctuations to depend on the stochastic trend. Our applications show that tests for seasonal unit roots in nonperiodic models may too often detect such roots, while, when allowing for periodically varying AR parameters, the evidence for seasonal unit roots tends to disappear.

One drawback of periodic autoregressions is that the number of parameters increases with the seasonal frequency. Hence, a periodic AR model for monthly time series can involve a huge number of parameters. This would complicate the empirical application of our approach, which seems to work well for quarterly data. To allow the application of our method to monthly data would then imply that one imposes restrictions on the various AR parameters. When such proper restrictions are imposed, the asymptotic results in our paper indicate that our selection strategy can easily be used.

\section{Appendix. Proofs of lemmas and theorems}

Proof of Lemma 3.1. The error term $\left\{\varepsilon_{T}\right\}$ is assumed Gaussian and hence satisfies the multivariate invariance principle, see, e.g. Phillips and Durlauf (1986), thus implying that

$$
\left(1 / N^{1 / 2}\right) \sum_{j=1}^{[r N]} \varepsilon_{j} \stackrel{\mathrm{d}}{\rightarrow} \sigma E(r)
$$

where $\sigma^{2} I_{4}=\lim _{N \rightarrow \infty} N^{-1} E\left(\left(\sum_{j=1}^{N} \varepsilon_{j}\right)\left(\sum_{j=1}^{N} \varepsilon_{j}\right)^{\prime}\right)$ is the covariance matrix of the vector standard Brownian motion $E(r)$ of dimension $(4 \times 1)$. It follows from the analysis of, e.g. Park and Phillips (1989) and Haldrup (1994a, b) that given (26)

$$
\begin{aligned}
\left(1 / N^{3 / 2}\right) Y_{[r N]}= & \left(1 / N^{3 / 2}\right)\left(\Theta_{0}+c: \Theta_{0}^{*}+\Theta_{1}^{*}\right) \Lambda(1)^{-1} \sum_{k=1}^{[r N 1} \sum_{j=1}^{k} \varepsilon_{j}+o_{p}(1) \\
& \stackrel{\mathrm{d}}{\rightarrow} \sigma\left(\Theta_{0}+\Theta_{1}\right)\left(\Theta_{0}^{*}+\Theta_{1}^{*}\right) \Lambda(1)^{-1} \int_{0}^{r} E(u) \mathrm{d} u \equiv \bar{B}(r) .
\end{aligned}
$$

It is now a consequence of the results in Boswijk and Franses (1996) that due to the unit roots in the VMA polynomial (24), the matrices $\left(\Theta_{0}+\theta_{1}\right)$ and $\left(\Theta_{0}^{*}+\Theta_{1}^{*}\right)$ both will be of rank 1 and

$$
\left(\Theta_{0}+\Theta_{1}\right)\left(\Theta_{0}^{*}+\Theta_{1}^{*}\right)=a b^{\prime} a^{*} b^{* \prime}
$$


where the $a, b, a^{*}$ and $b^{*}$ vectors are given in (29). Define now the scalar integrated standard Brownian motion

$$
\bar{W}(r)=\sigma \omega^{-1} b^{\prime} a^{*} b^{* \prime} \Lambda(1)^{-1} \int_{0}^{r} E(u) \mathrm{d} u,
$$

with $w$ given in (28) and the first part of (27) follows straightforwardly. With respect to the second part of (27) it follows from

$$
\Delta Y_{T}^{*}=\left(\Theta_{0}^{*}+\Theta_{1}^{*} B\right) A(B)^{-1} \varepsilon_{T}
$$

that

$$
\begin{aligned}
\left(1 / N^{1 / 2}\right) Y_{[, N]}^{*}= & \left(1 / N^{1 / 2}\right)\left(\Theta_{0}^{*}+\theta_{1}^{*} B\right) A(B)^{-1} \sum_{j=1}^{[N, N]} \varepsilon_{j}+o_{p}(1) \\
& \stackrel{d}{\rightarrow} \sigma\left(\Theta_{0}^{*}+\Theta_{1}^{*}\right) A(1)^{-1} E(r) \equiv B^{*}(r) .
\end{aligned}
$$

Similarly to the analysis in Boswijk and Franses (1996) and the procedure above, we now have that

$$
R^{*}(r)=\sigma a^{*} b^{* \prime} A(1)^{-1} E(r),
$$

and with the standard Brownian motion

$$
W(r)=\sigma \omega^{*-1} b^{* \prime} A(1)^{-1} E(r),
$$

the required result follows.

Proof of Theorem 3.1. We shall consider model (30) both under the restriction $\alpha_{1} \alpha_{2} \alpha_{3} \alpha_{4}=1$ and the additional restriction $\beta_{1} \beta_{2} \beta_{3} \beta_{4}=1$. Our setup encompasses both situations. For the latter case, we write the model as $y_{t}=x_{t}(\gamma)+\varepsilon_{t}$, where $\gamma=\left(\alpha_{2}, \alpha_{3}, \alpha_{4}, \beta_{2}, \beta_{3}, \beta_{4}\right)^{\prime}$, which we also may write in the condensed form $\gamma=\left(\alpha^{\prime}, \beta^{\prime}\right)^{\prime}$. When the restriction $\beta_{1} \beta_{2} \beta_{3} \beta_{4}=1$ is not imposed in the estimation, the $\gamma$ vector may be redefined such that $\beta$ is simply $\left(\beta_{1}, \beta_{2}, \beta_{3}, \beta_{4}\right)^{\prime}$. Define the vector of pseudo regressors

$$
\partial x_{t} / \partial y=\left(z_{1}^{\prime}, w_{t}^{\prime}\right)^{\prime},
$$

where $z_{t}=J_{1}^{\prime} v_{t}, w_{t}=J_{2}^{\prime} u_{t}$ with $v_{t}=\left(v_{1, t}, v_{2, t}, v_{3, t}, v_{4, t}\right), u_{t}=\left(u_{1, t}, u_{2, t}, u_{3, t}\right.$, $\left.u_{4, t}\right)^{t}, v_{s, t}=D_{s, t} y_{t-1}-\beta_{s+1} D_{s+1, t} y_{t-2}, u_{s, t}=D_{s, t}\left(y_{t-1}-\alpha_{s-1} y_{t-2}\right)$ and with $J_{1}$ and $J_{2}$ defined as

$$
J_{1}^{\prime}=\left[\begin{array}{cccc}
-1 /\left(\alpha_{2}^{2} \alpha_{3} \alpha_{4}\right) & 1 & 0 & 0 \\
-1 /\left(\alpha_{2} \alpha_{3}^{2} \alpha_{4}\right) & 0 & 1 & 0 \\
-1 /\left(\alpha_{2} \alpha_{3} \alpha_{4}^{2}\right) & 0 & 0 & 1
\end{array}\right]
$$


and

$$
J_{2}^{\prime}=\left[\begin{array}{cccc}
-1 /\left(\beta_{2}^{2} \beta_{3} \beta_{4}\right) & 1 & 0 & 0 \\
-1 /\left(\beta_{2} \beta_{3}^{2} \beta_{4}\right) & 0 & 1 & 0 \\
-1 /\left(\beta_{2} \beta_{3} \beta_{4}^{2}\right) & 0 & 0 & 1
\end{array}\right],
$$

respectively. When the restriction $\beta_{1} \beta_{2} \beta_{3} \beta_{4}=1$ is not imposed when estimating the parameters, all the derived results will carry through with $J_{2}$ substituted by a $(4 \times 4)$ identity matrix. Notice that we also let $\alpha_{1}=\left(\alpha_{2} \alpha_{3} \alpha_{4}\right)^{-1}$ and $\beta_{1}=\left(\beta_{2} \beta_{3} \beta_{4}\right)^{-1}$. Furthermore, define $\alpha_{0}=\alpha_{4}, \beta_{0}=\beta_{4}$. Hence, we have that the NLS estimates can be found asymptotically as

$$
\left[\begin{array}{l}
\hat{\alpha}-\alpha \\
\hat{\beta}-\beta
\end{array}\right]=\left[\begin{array}{llll}
\sum_{t=1}^{n} & z_{t} z_{l}^{r} & \sum_{t=1}^{n} & z_{t} w_{l}^{\prime} \\
\sum_{t=1}^{n} & w_{t} z_{t}^{\prime} & \sum_{t=1}^{n} & w_{t} w_{t}^{\prime}
\end{array}\right]^{-1}\left[\begin{array}{ll}
\sum_{t=1}^{n} z_{t} \eta_{t} \\
\sum_{t=1}^{n} w_{t} \eta_{t}
\end{array}\right] .
$$

To derive the order of $\hat{\alpha}$ and $\hat{\beta}$ we require that after appropriate normalization the diagonal submatrices $\sum_{i=1}^{n} z_{\mathrm{r}} z_{t}^{\prime}$ and $\sum_{i=1}^{n} w_{1} w_{1}^{\prime}$ are nonsingular. To show this we write (A.9) in the following way:

$$
\begin{aligned}
& {\left[\begin{array}{l}
\hat{\alpha}-\alpha \\
\hat{\beta}-\beta
\end{array}\right]=\left[\left[\begin{array}{ll}
J_{1}^{\prime} & 0 \\
0 & J_{2}^{\prime}
\end{array}\right]\left[\begin{array}{lll}
\sum_{t=1}^{n} & v_{t} v_{t}^{\prime} & \sum_{t=1}^{n} v_{t} u_{t}^{\prime} \\
\sum_{t=1}^{n} & u_{t} v_{t}^{\prime} & \sum_{t=1}^{n} u_{t} u_{t}^{\prime}
\end{array}\right]\left[\begin{array}{ll}
J_{1}^{\prime} & 0 \\
0 & J_{2}^{\prime}
\end{array}\right]\right]^{-1}} \\
& \times\left[\begin{array}{ll}
J_{1} & 0 \\
0 & J_{2}
\end{array}\right]\left[\begin{array}{ll}
\sum_{\mathrm{r}=1}^{\mathrm{n}} & v_{\mathrm{t}} \eta_{\mathrm{t}} \\
\sum_{\mathrm{t}=1}^{\mathrm{n}} & u_{\mathrm{r}} \eta_{t}
\end{array}\right] \text {. }
\end{aligned}
$$

Along the lines of Boswijk and Franses (1996) we let $V_{s . T}$ indicate the VQ process of $v_{s, 1-}$ This is a different process for each $s$. If the $(4 \times 4)$ matrix $\Psi_{(B)}$ defined in (12) is partitioned such that row number $s$ is $\psi_{s}$, it follows that

$$
V_{s, T}=\psi_{s}(B) Y_{s-1, T}=\psi_{s} Y_{s-1 . T}+\psi_{s}^{*}(B) \Delta_{1} Y_{s-1, T},
$$

where $\psi_{s}^{*}(B)$ follows from a polynomial decomposition of $\psi_{s}(B)$. Since the term $\psi_{s} Y_{s-1, T}$ is doubly integrated whilst $\Delta_{1} Y_{s-1 . r}$ is integrated of order one, it follows from Park and Phillips (1989) and Haldrup (1994a) that

$$
\begin{aligned}
N^{-4} \sum_{t=1}^{n} v_{s, t} v_{q, t}= & N^{-4} \sum_{T=1}^{N} V_{s, T}^{\prime} V_{q, T}=\left(\psi_{s} \psi_{q}\right)\left(N^{-4}\right) \\
& \times \sum_{T=1}^{N} Y_{s-1 . T} Y_{q-1 . T}+o_{p}(1)
\end{aligned}
$$

for $s, q=1,2,3,4$. In accordance with Lemma 3.1, we therefore have that

$$
N^{-4} \sum_{i=1}^{n} v_{r} v_{t}^{\prime} \stackrel{\mathrm{d}}{\rightarrow} \omega^{2} A \Psi^{\prime} \Psi_{A} \int_{0}^{1}(\bar{W}(\mathrm{r}))^{2} \mathrm{~d} r
$$

where $A=\operatorname{diag}\left(a_{4}, a_{1}, a_{2}, a_{3}\right)$. In a similar fashion the VQ process corresponding to $u_{s, t}$ reads

$$
U_{s, T}=\psi_{s}^{*} Y_{s-1 . T}^{*},
$$


where $\Psi^{*}=I_{4}$ and $\psi_{s}^{*}$ corresponds to column $s$ of this matrix. Because the columns are orthogonal, we have that the expression

$$
N^{-2} \sum_{t=1}^{n} u_{s, r} u_{q, t}=N^{-2} \sum_{r=1}^{N} U_{s, r}^{\prime} U_{q, T}^{\prime}
$$

is 0 for $s \neq q$, while for $s=q$ the expression yıelds

$$
N^{-2} \sum_{t=1}^{n} u_{s, t}^{2}=N^{-2} \sum_{T=1}^{N} Y_{s-1, T}^{*} Y_{s-1 . T}^{*}
$$

It follows in accordance with Lemma 3.1 that

$$
N^{-2} \sum_{i=1}^{n} u_{1} u_{1}^{\prime}=\omega^{* 2} A^{*} A^{*} \int_{0}^{1} W(r)^{2} \mathrm{~d} r
$$

where $A^{*}=\operatorname{diag}\left(a_{4}^{*}, a_{1}^{*}, a_{2}^{*}, a_{3}^{*}\right)$.

The nonsingularity conditions that have to be met require that the matrices $J_{1}^{\prime} A \Psi^{\prime}$ and $J_{2}^{\prime} A^{*}$ are of full rank equal to 3 . Both $J_{1}^{\prime}$ and $J_{2}^{\prime}$, which are $(3 \times 4)$ matrices, are of full rank by their construction. When the restriction on the $\beta$ 's is not imposed, $J_{2}$ is naturally full rank of dimension $(4 \times 4)$. $A$ and $A^{*}$ are full rank matrices whilst $\Psi$ is of rank 3 . It follows that $J_{2}^{x} A^{*}$ is full rank and it also follows from the construction of $J_{1}$ that the first rank condition will be satisfied. To conclude, the matrices $N^{-4} \sum_{t=1}^{n} z_{1} z_{t}^{\prime}$ and $N^{-4} \sum_{t=1}^{n} w_{t} w_{t}^{\prime}$ are indeed nonsingular.

Now, define $D_{k}=\operatorname{diag}\left(N^{3 / 2} I_{3}, N^{1 / 2} I_{3}\right)$. It can be deduced from Haldrup (1994a) that in this case

$$
\begin{aligned}
N^{1 / 2} D_{N}\left[\begin{array}{c}
\hat{\alpha}-\alpha \\
\hat{\beta}-\beta
\end{array}\right]= & {\left[N^{-1} D_{N}^{-1}\left[\begin{array}{ccc}
\sum_{\mathrm{t}=1}^{n} z_{t} z_{t}^{\prime} & \sum_{t=1}^{n} z_{t} w_{t}^{\prime} \\
\sum_{t=1}^{n} & w_{t} z_{t}^{\prime} & \sum_{t=1}^{n} w_{t} w_{t}^{\prime}
\end{array}\right] D_{N}^{-1}\right]^{-1} } \\
& \times N^{-1 i^{2}} D_{N}^{-1}\left[\begin{array}{ll}
\sum_{t=1}^{n} & z_{t} \eta_{t} \\
\sum_{t=1}^{n} & w_{t} \eta_{t}
\end{array}\right]=O_{\mathrm{p}}(1) .
\end{aligned}
$$

Therefore,

$$
N^{3}(\hat{\alpha}-\alpha)=\mathrm{O}_{\mathrm{p}}(1), \quad N(\hat{\beta}-\beta)=\mathrm{O}_{\mathrm{p}}(1) .
$$

Because $\alpha_{1}=\left(\alpha_{2} \alpha_{3} \alpha_{4}\right)^{-1}$, we also have that

$$
N^{2}\left(\hat{\alpha}_{1}-\alpha_{1}\right)=-\left(\alpha_{2} \alpha_{3} \alpha_{4}\right)^{-1} \sum_{s=2}^{4}\left(\alpha_{s}\right)^{-1} N^{2}\left(\hat{\alpha}_{s}-\alpha_{s}\right)=O_{p}(1) \text {. }
$$

A similar argument applies to $N\left(\hat{\beta}_{1}-\beta_{1}\right)$. Notice that these results will apply regardless of imposing the restriction $\beta_{1} \beta_{2} \beta_{3} \beta_{4}=1$ in the NLS estimation.

Proof of Theorem 4.I. Consider the general VQ representation of the PAR $(p)$ model

$$
\Phi_{0} Y_{T}=\Phi_{1} Y_{T-1}+\cdots+\Phi_{P} Y_{T-P}+\varepsilon_{T}, \quad \varepsilon_{T} \sim \mathrm{IN}(0, \Sigma), \quad T=1, \ldots, N
$$


where $P$ is an integer obeying $[(p-1) / 4]+1$, where $[-]$ means 'integer of', where $\Sigma=\sigma^{2} I_{4}$ and

$$
\Phi_{0}=\left[\begin{array}{ccrc}
1 & 0 & 0 & 0 \\
-\phi_{12} & 1 & 0 & 0 \\
-\phi_{23} & -\phi_{13} & 1 & 0 \\
-\phi_{34} & -\phi_{24} & -\phi_{14} & 1
\end{array}\right], \quad\left(\Phi_{i}\right)_{s j}=\phi_{(4 i+s m j) . s}, \quad i=1, \ldots, P
$$

In error correction form, the model reads as

$$
\begin{aligned}
& \Delta Y_{T}=\Pi Y_{T-1}+\Gamma_{1} \Delta Y_{T-1}+\cdots+\Gamma_{P-1} \Delta Y_{T-P+1}+v_{T}, \\
& v_{T} \sim \mathrm{IN}(0, \Omega)
\end{aligned}
$$

where $v_{T}=\Phi_{0}^{-1} \varepsilon_{T}$, where $\Omega=\Phi_{0}^{-1} \Sigma \Phi_{0}^{-1 \prime}=\sigma^{2}\left(\Phi_{0}^{\prime} \Phi_{0}\right)^{-1}$, and

$$
\begin{aligned}
& \Pi=-\Phi_{0}^{-1}\left(\Phi_{o}-\cdots-\Phi_{p}\right), \quad \Gamma_{i}=-\Phi_{0}^{-1}\left(\Phi_{i+1}+\cdots+\Phi_{P}\right), \\
& i=1, \ldots, P-1 .
\end{aligned}
$$

The null hypothesis of $(4-r)$ unit roots expressed as

$$
\mathbf{H}_{\mathbf{0}}: \quad \Pi=\gamma \alpha^{\prime},
$$

where the matrix $\alpha$ is defined in Section 4 for $r=1,2,3$; for $r=0$, take $\alpha$ to be equal to 0 . Define the matrix $\Xi=\Pi-\gamma \alpha^{\prime}$, so that one way to express the model is

$$
\begin{aligned}
& \Delta Y_{\mathrm{T}}=\gamma \alpha^{\prime} Y_{T-1}+\Xi Y_{T-1}+\Gamma_{1} \Delta Y_{T-1}+\cdots+\Gamma_{P-1} \Delta Y_{T-P+1}+v_{T}, \\
& v_{T} \sim \mathrm{IN}(0, \Omega),
\end{aligned}
$$

and the null hypothesis becomes $H_{4}: \Xi=0$.

Suppose that we disregard the restrictions imposed upon (A.15) by the underlying periodic model. Define the full parameter vector $\theta=\left(\theta_{1}^{r}, \theta_{2}, \theta_{3}^{\prime}\right)^{\prime}$, where $\theta_{\mathrm{I}}$ contains the free parameters in $\left(\gamma, \Gamma_{1}, \ldots, \Gamma_{\mathrm{P}-1}, \Omega\right)$, and where $\theta_{2}$ and $\theta_{3}$ contain the identified parameters in $\alpha$ and $\Xi$, respectively. The dimension of $\theta_{1}$ is $4 r+16(P-1)+10$. From Johansen's (1991) results it can be seen that the dimension of $\theta_{2}$ is $r(4-r)$, and from this it can in turn be deduced that $\operatorname{dim} \theta_{3}$ equals $(4-r)^{2}$.

The periodic model however implies certain over-identifying restrictions on (A.15). Without the unit root restrictions, the total number of free parameters is $4 p+1$. With $(4-r)$ unit roots, this number is $4(p-(4-r))+I+r(4-r)$, so that this hypothesis corresponds to $(4-r)^{2}$ restrictions, which corresponds exactly with the dimension of $\theta_{3}$. Likewise, from Section 4 it can be seen that the dimension of the vector of parameters in the cointegrating vectors is $r(4-r)$, which corresponds to the dimension of $\theta_{2}$ in the unrestricted VQ model. Thus, the Inderlying periodic structure implies only restrictions on $\theta_{1}$, i.e. on the 
'short-run' parameters (in the sensc that these do not characterize unit roots or cointegrating vectors). Let us express these restrictions as $g\left(\theta_{1}\right)=0$. From the above, it follows that the dimension of the vector function $g$ equals $16 P-4 p+9$, which is exactly equal to the difference in the number of parameters between a four-dimensional VAR $(P)$ and a univariate $\operatorname{PAR}(p)$ process.

Let $\Theta=\Theta_{1} \times \Theta_{2} \times \Theta_{3}$ denote the unrestricted parameter space, where $\Theta_{i}$ is the parameter space for $\theta_{i}, i=1,2,3$. Next, let $\Theta_{1}^{p}=\left\{\theta_{1} \in \Theta_{1}: g\left(\theta_{1}\right)=0\right\}$, the restricted parameter space corresponding to the periodic structure, and let $\Theta_{0}^{r}=\{0\}$, the restricted parameter space corresponding to the hypothesis of $(4-\mu)$ unit roots. Similarly, define

$$
\begin{aligned}
& \Theta^{p}=\Theta_{1}^{p} \times \Theta_{2} \times \Theta_{3}, \quad \Theta^{r}=\Theta_{1} \times \Theta_{2} \times \Theta_{3}^{r}, \\
& \Theta^{p r}=\Theta^{p} \cap \Theta^{r}=\Theta_{1}^{p} \times \Theta_{2} \times \Theta_{3}^{r} .
\end{aligned}
$$

Johansen's likelihood ratio statisti: for the hypothesis of $(4-r)$ unit roots in the unrestricted VAR may be expressed as

$$
\operatorname{LR}\left(\Theta^{r} \mid \Theta\right)=-2\left(\max _{\theta \subset \theta^{r}} \log L(\theta)-\operatorname{mix}_{\theta} x_{\theta} \log L(\theta)\right),
$$

with $L(\theta)$ the likelihood function. On the other hand, the LR statistic for ( $4-r)$ unit roots in the periodic autoregression is given by

$$
\mathbf{L R}\left(\Theta^{p r} \mid \Theta^{p}\right)=\mathbf{L R}\left(\Theta^{r} \mid \Theta\right)+\mathbf{L R}\left(\Theta^{p r} \mid \Theta^{r}\right)-\operatorname{LR}\left(\Theta^{p} \mid \Theta\right) .
$$

Note that the last two terms on the right-hand side are the likelihood ratio statistics for the restriction $g\left(\theta_{1}\right)=0$, with and without the unit roots imposed. Slightly extending the results of Johansen (1991, Appendix C), it can be shown that $\left(N^{1 / 2}\right)\left(\hat{\theta}_{1}-\theta_{1}\right)$ is asymptotically independent from $N\left(\hat{\theta}_{2}-\theta_{2}\right)$ and $N\left(\hat{\theta}_{3}-\theta_{3}\right)$. This implies that

$$
\operatorname{LR}\left(\Theta^{p r} \mid \Theta^{p}\right)-\operatorname{LR}\left(\Theta^{r} \mid \Theta\right)=\operatorname{LR}\left(\Theta^{r r} \mid \Theta^{r}\right)-\operatorname{LR}\left(\Theta^{p} \mid \Theta\right) \stackrel{P}{\rightarrow} 0,
$$

so that the LR statistic for $(4-r)$ unit roots in the Feriodic autoregression is asymptotically equivalent to the LR statistic for $(4-r)$ unit roots in the unrestricted VAR model. Hence,

$$
\begin{aligned}
\operatorname{LR}\left(\Theta^{p r} \mid \Theta^{p}\right) \stackrel{\mathrm{d}}{\rightarrow} & \text { trace }\left\{\int _ { 0 } ^ { 1 } \left(\mathrm{d} W(s) W(s)^{\prime}\right.\right. \\
& \left.\times\left(\int_{0}^{1} W(s) W(s)^{\prime} \mathrm{d} s\right)^{-1} \int_{0}^{1} W(s) \mathrm{d} W(s)^{r}\right\},
\end{aligned}
$$

where $W(s)$ is a standard(4-r)-dimensional Brownian motion process, see Johansen (1991). Quantiles of this distribution are tabulated in OsterwaldLenum (1992, Table 0). Extensions to fitted intercepts and linear trends 
can be proved analogously; see Osterwald-Lenum (1992) for tables with the relevant critical values.

In a similar fashion, we can prove that with the correct number of unit roots imposed, the likelihood ratio statistic for restrictions on $\alpha$ has an asymptotic $\chi^{2}$ distribution under the null hypothesis, whether or not the restriction $g\left(\theta_{1}\right)=0$ is imposed. Let us denote such restrictions on $\alpha$ by $h\left(\theta_{2}\right)=0$, and the corresponding restricted parameter space of $\theta_{2}$ by $\Theta_{2}^{h}=\left\{\theta_{2} \in \Theta_{2}: h\left(\theta_{2}\right)=0\right\}$. Likewise, define $\Theta^{p h r}=\Theta_{1}^{p} \times \Theta_{2}^{h} \times \Theta_{3}^{r}$, and so on. Then, the likelihood ratio statistic for the restrictions on $\theta_{2}$ in the periodically integrated AR model satisfies

$$
\begin{aligned}
\operatorname{LR}\left(\Theta^{p h r} \mid \Theta^{p r}\right) & =\operatorname{LR}\left(\Theta^{h r} \mid \Theta^{r}\right)+\operatorname{LR}\left(\Theta^{p h r} \mid \Theta^{h r}\right)-\operatorname{LR}\left(\Theta^{p r} \mid \Theta^{r}\right) \\
& =\operatorname{LR}\left(\Theta^{h r} \mid \Theta^{r}\right)+o_{p}(1) \stackrel{d}{\rightarrow} \chi^{2}(m),
\end{aligned}
$$

where $m$ is the dimension of $h$. The second equality follows from the fact that the LR statistic for the restrictions on $\theta_{1}$ is independent of whether restrictions have been imposed on $\alpha$; the limiting $\chi^{2}$ distribution is proved in Johansen (1991, Appendix C).

\section{References}

Boswijk, H.P., Franses, P.H., 1996. Unit roots in periodic autoregressions. Journal of Time Series Analysis 17, 221-245.

Franses, P.H., 1994. A Multivariate approach to modeling univariate seasonal time series. Journal of Econometrics 63, 133-15t.

Franses, P.H., Paap, R., 1994. Model selection in periodic autoregressions. Oxford Bulletin of Economics and Statistics $56,421-439$.

Franses, P.H., Romijn, G., 1993. Periodic integration in quarterly UK macroeconomic variables, International Journal of Forecasting 9, 467-476.

Ghyscls, E., Hall, A., Lee, H.S., 1996. On periodic structures and testing for scasonal unit roots. Journal of the American Statistical Association, 91, 1551-1559.

Haldrup, N., 1994a. The asymptotics of single equation cointegration regression models with I(1) and $1(2)$ variables. Journal of Econometrics 63, 153-181.

Haldrup, N., 1994b. Semi-parametric tests for doubie unit roots. Journal of Business and Economic Statistics $12,109-122$.

Hylleberg, S., Engle, R.F., Granger, C.W.J., Yoo, B.S., 1990. Seasonal integration and cointegration. Journal of Econometrics 44, 21 S-238.

Johansen, S., 1988. Statistical analysis of cointegration vectors. Journal of Economic Dynamics and Control 12, 113-125.

Johansen, S., 1991. Estimation and hypothesis testing of cointegration vectors in Gaussian vector auteregressive models. Econometrica 59, 1551-1580.

Johansen, S., 1992a. A representation of vector autorcgressive processes integrated of order 2 . Econometric Theory 8, 188-202.

Johansen, S., 1992b. Determination of cointegration rank in the presence of a linear trend. Oxford Bulletin of Economics and Statistics 54, 383-397.

Lütkepoh], H., 1991. Introduction to Multiple Time Serjes Analysis, Springer, Berlin. 
Osborn, D.R., 1988. Seasonality and habit persistence in a life-cycle model of consumption. Journal of Applied Econometrics 3, 255-266.

Osterwald-Lenum, M., 1992. A note with quantiles of the asymptotic distribution of the maximum likelihood cointegration rank test statistics: four cases. Oxford Bulletin of Economics and Statistics 54, 461-471.

Pantula. S.G., 1989. Testing for unit roots in time series data. Econometric Theory 5, 256-271.

Park, J.Y., Phillips, P.C.B., 1989. Statistical inference in regressions with integrated processes: Part 2. Econometric Theory 5, 95-131.

Phillips, P.C.B., Durlauf, S.FN., 1986. Multiple time series regression with integrated processes. Review of Economic Studies 53, 473-495.

Tiao, G.C..Grupe, M.R., 1980. Hidden periodic autoregressive-moving average models in time series data. Biometrika 67, 365-373. 